\title{
Study of the coherent perturbation of a Michelson interferometer due to the return from a scattering surface
}

Vitalii Khodnevych, Sibilla Di Pace, Jean-Yves Vinet, Nicoleta Dinu-Jaeger and Michel Lintz

Laboratoire ARTEMIS, Universite Cote d'Azur, Observatoire de la Cote d'Azur and CNRS

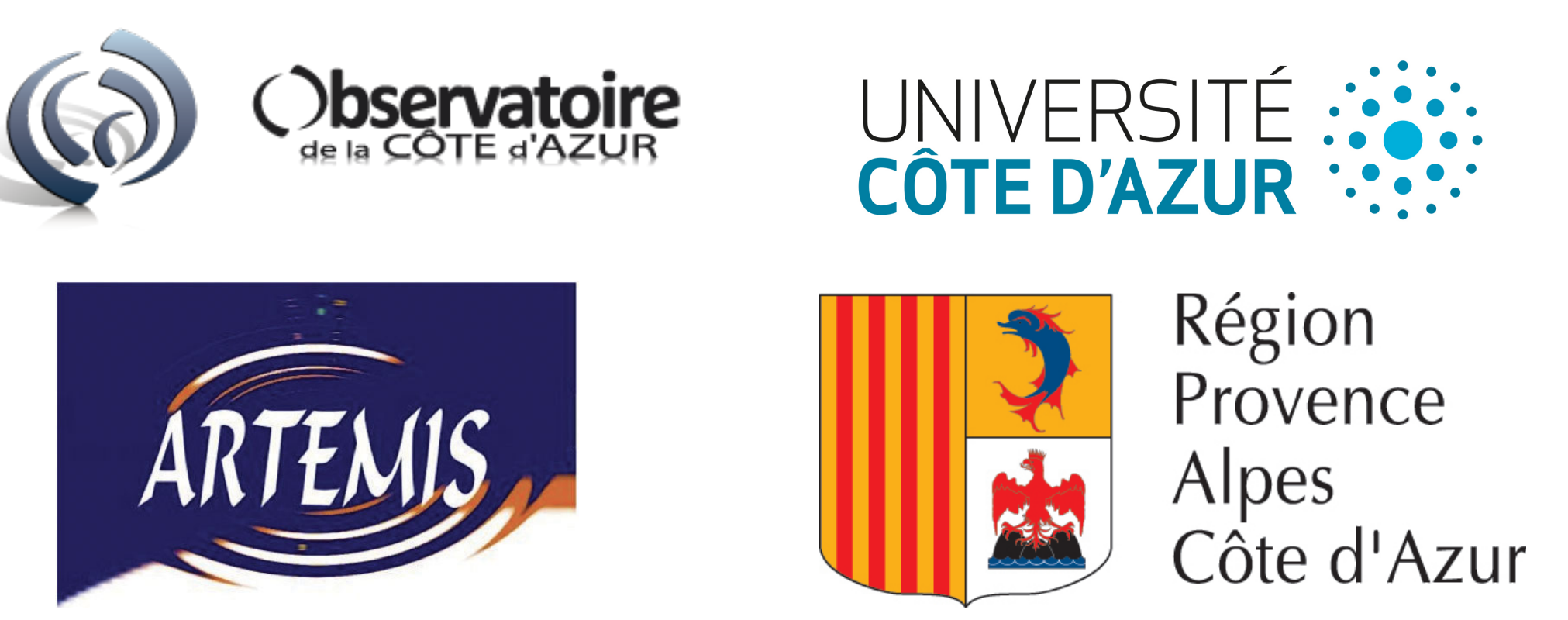

THALES

\begin{tabular}{|l|}
\hline Goals \\
- Build a setup for coherent measurements \\
of the backscattered light \\
- Based on Michelson interferometer \\
- Special data processing for elimination of \\
stray contributions \\
- Characterize dependence with: \\
$\quad$ - spot position \\
$\quad$ - incidence angle \\
- Study scattering by: \\
$\quad$ - surface roughness \\
- dust particles
\end{tabular}

\section{Data processing}

Special data processing was developed for the extraction of the useful signal from the various stray contributions to the coherent signal.

Multibeam interference:

$I_{A C}=\frac{I_{L}}{2}\left(\sum_{i} \sqrt{b_{i}} \cos \left(\Delta \phi_{i}\right)+\sqrt{b_{s}} \cos \left(\Delta \phi_{s}\right)\right)$

Demodulated amplitude:

$R_{1}=I_{L}\left(\sum_{i} \sqrt{b_{i}} J_{1}\left(2 \pi \delta_{P M i}\right) \cos \left(\phi_{i}^{t}\right)+\right.$

$\left.\sqrt{b_{s}} J_{1}\left(2 \pi \delta_{P M s}\right) \cos \left(\phi_{s}^{t}\right)\right)$

Slow modulation of the sample:

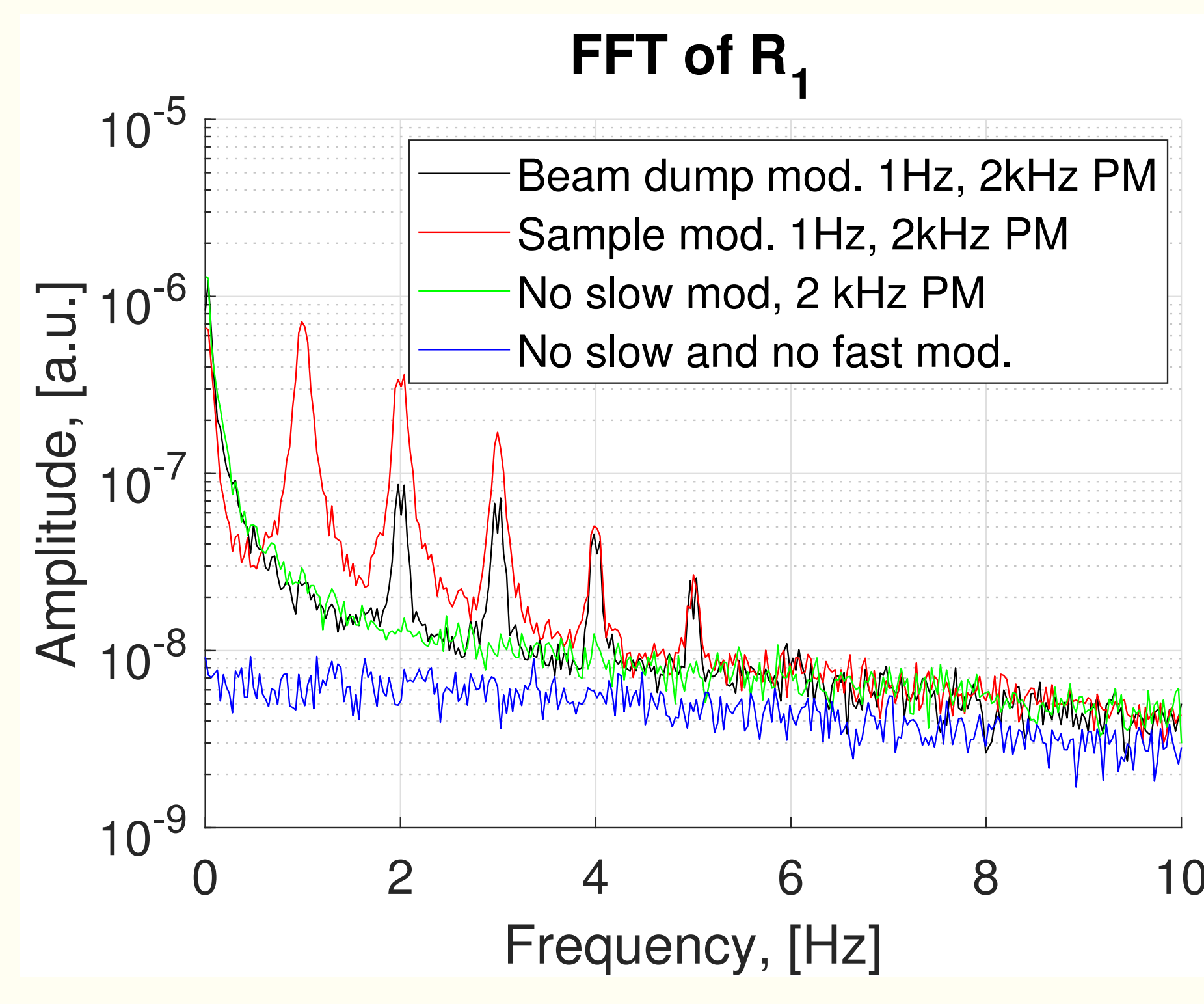

$R_{1}=I_{L}\left(\sum \sqrt{b_{i}} J_{1}\left(2 \pi \delta_{P M}\right) \cos \left(\phi_{i}^{t}\right)+\right.$

$$
\text { Stray }
$$

$\underbrace{\sqrt{b_{s}} J_{1}\left(2 \pi \delta_{P M}\right) \cos \left(2 \pi \delta_{1} \cos (2 \pi(1 H z) t)+\phi_{0 s}^{t}\right)}$ Sample

$\underbrace{\sqrt{b_{B D}} J_{1}\left(2 \pi \delta_{P M}\right) \cos \left(4 \pi \delta_{1} \cos (2 \pi(1 H z) t)+\phi_{0}^{t}\right)})$ Beam dump

after adjustment $\sqrt{b_{B D}}=0$ and $J_{0}\left(2 \pi \delta_{1}\right)=0$, so:

$$
\begin{aligned}
& R_{1}=I_{L} \sqrt{b_{s}} J_{1}\left(2 \pi \delta_{P M}\right) \cos \left(\phi_{s}\right) \\
& R_{2}=I_{L} \sqrt{b_{s}} J_{2}\left(2 \pi \delta_{P M}\right) \sin \left(\phi_{s}\right)
\end{aligned}
$$

combination gives backscattering in amplitude:

$\sqrt{b_{s}}=\sqrt{\left(\frac{R_{1}}{2 J_{1}\left(2 \pi \delta_{P M}\right)}\right)^{2}+\left(\frac{R_{2}}{2 J_{2}\left(2 \pi \delta_{P M}\right)}\right)^{2}} \frac{2}{I_{L}}$

\section{Acknowledgements}

This work is co-funded by: Region PACA, Thales Alenia Space, CNES, CNRS.

\section{Setup}

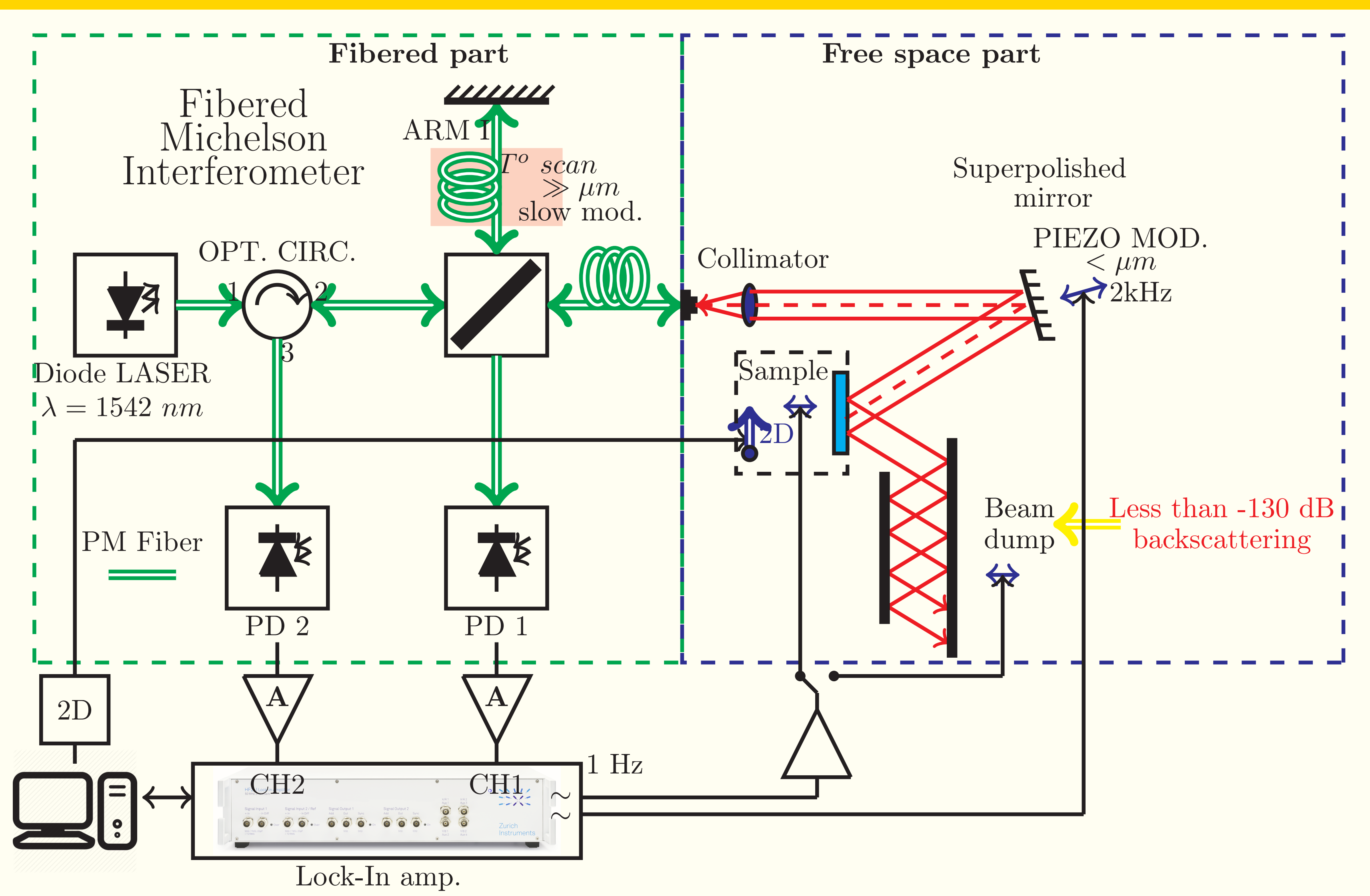

Figure 1: Schematics of coherent backscattering measurement setup. Green: PM optical fibers. Red: free space beams. Dashed red: backscattered light from the sample.

\section{Results of the XY and the angular scans}

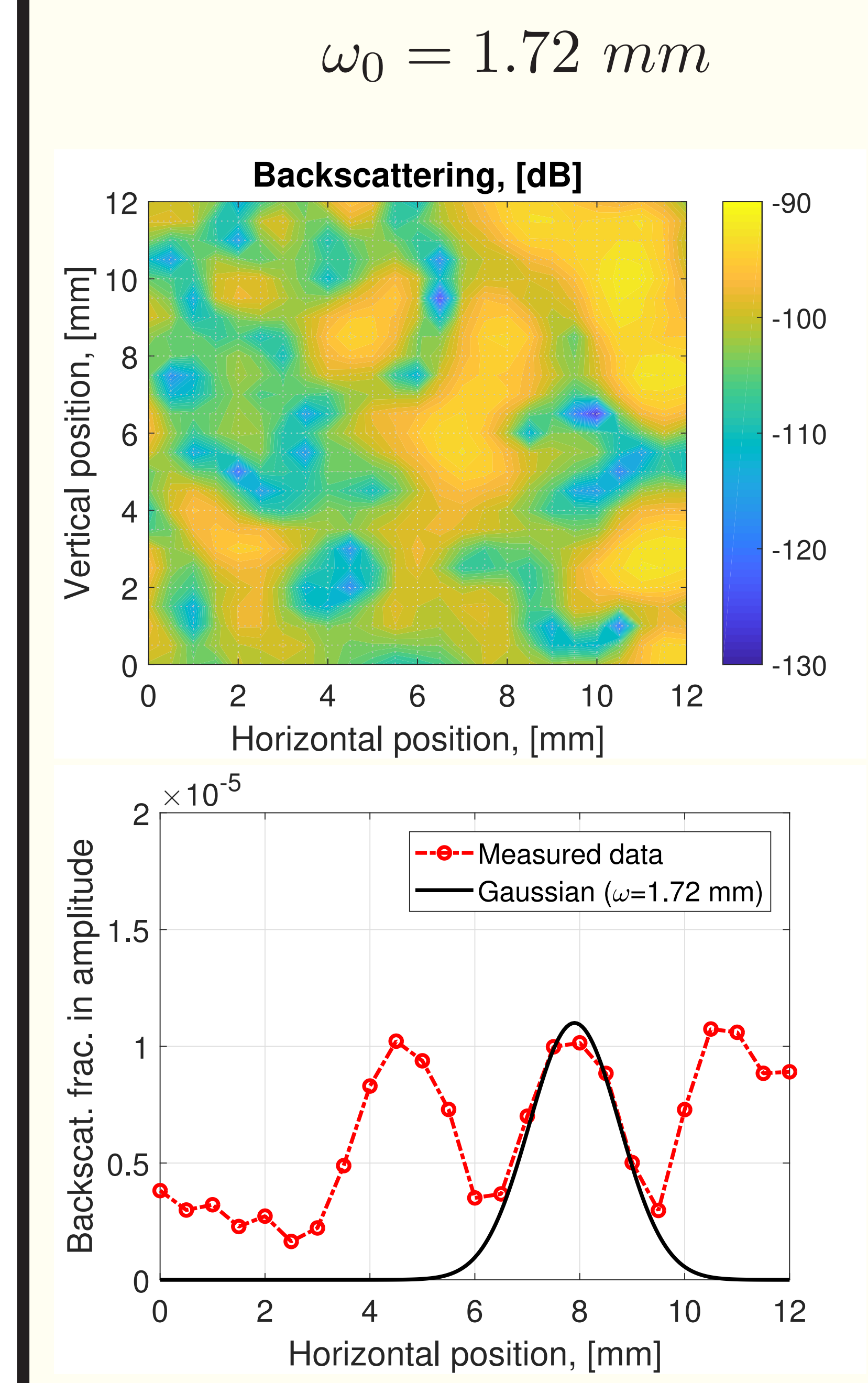

$\omega_{0}=0.65 \mathrm{~mm}$

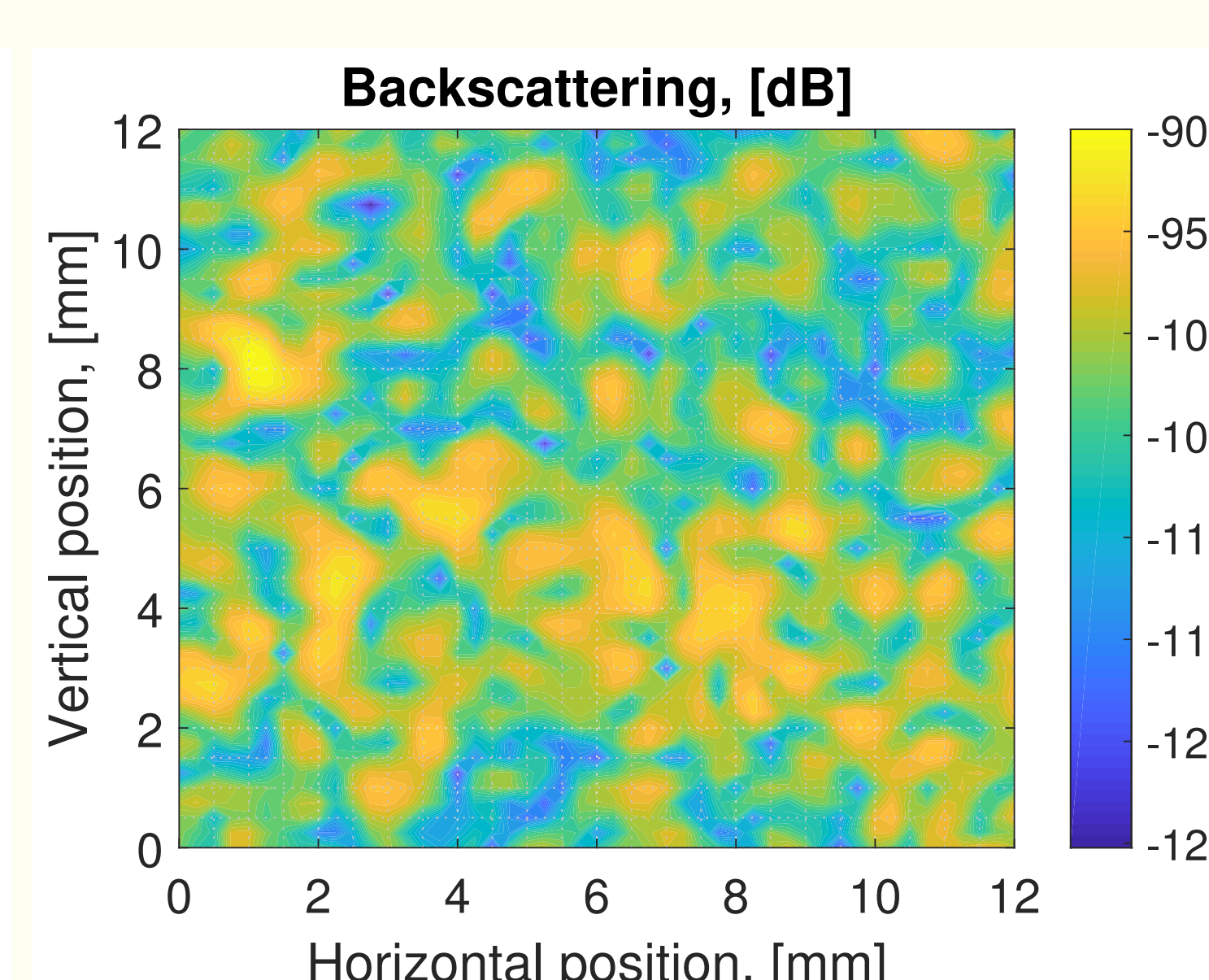

Horizontal position, $[\mathrm{mm}]$

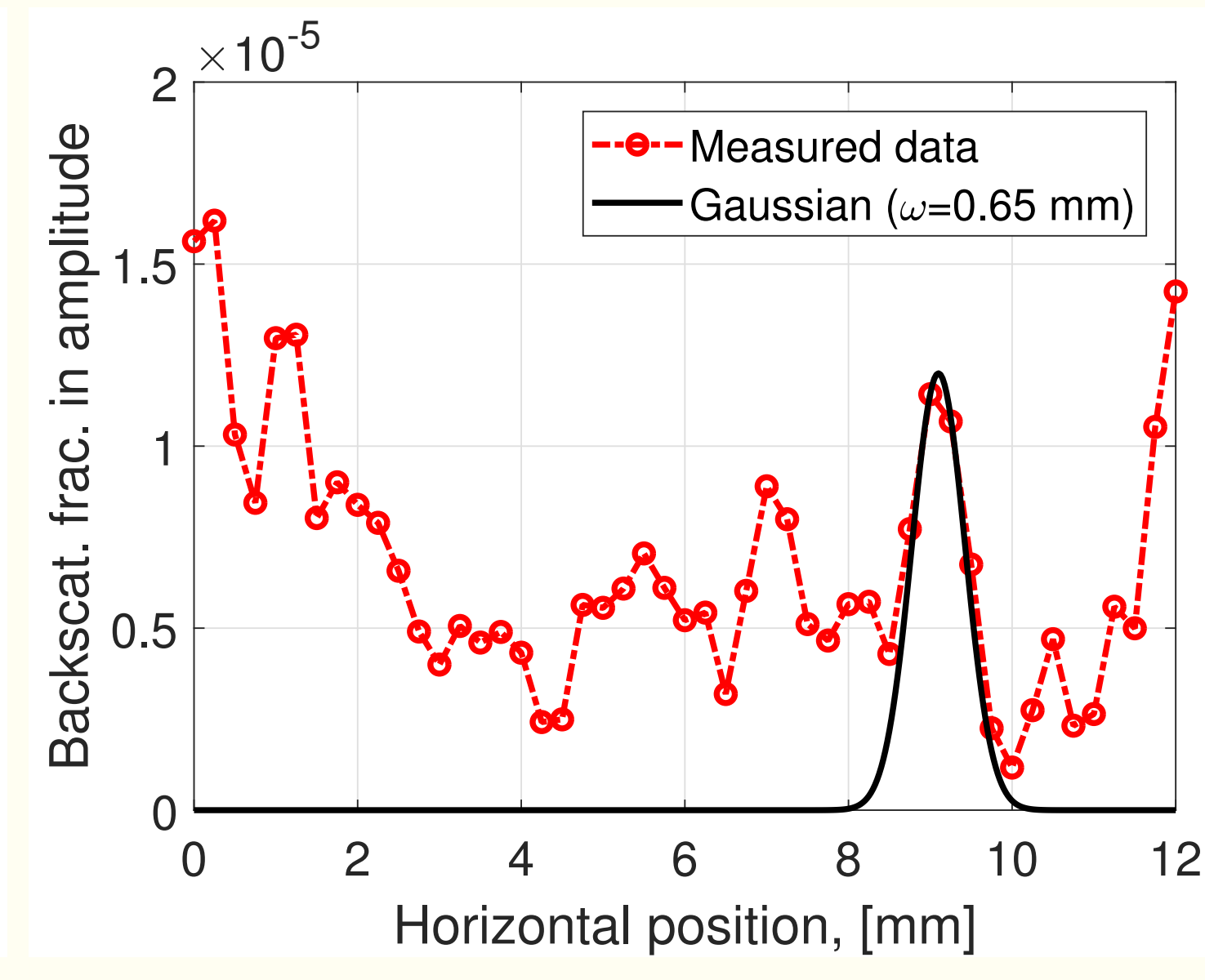

We have studied scattered light from an IR mirror with intermediate roughness (>175 A rms roughness). We have observed, in the coherent backscattering amplitude, a speckle-type dependence with the spot position and the incidence angle, as can be expected from the stochastic nature of the roughness profile.

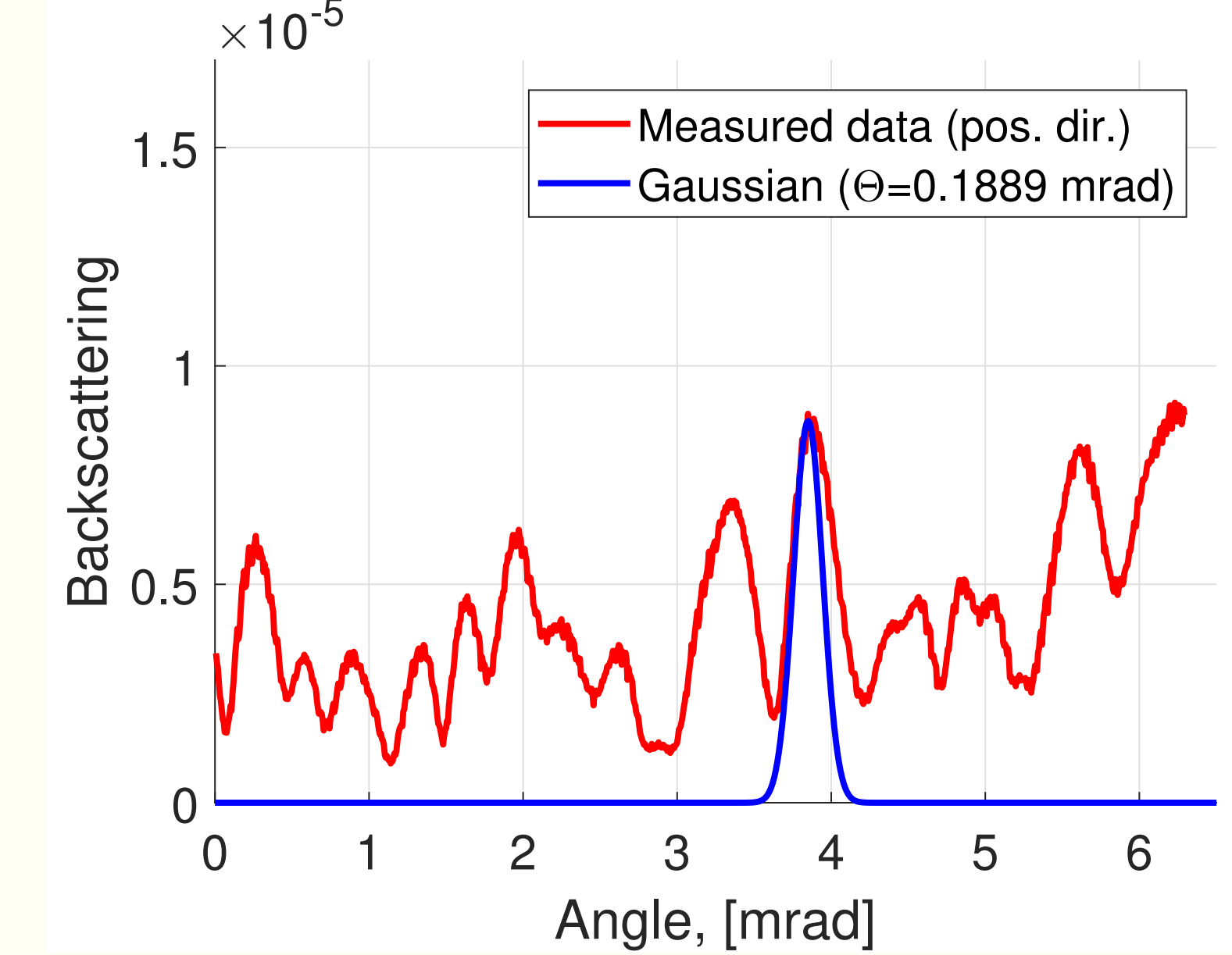

Figure 2: Size of speckle grain corresponds to the size

Figure 3: Typical angular size of speckle grain is $1 / 4$ of the beam divergence

\section{Polarization study}
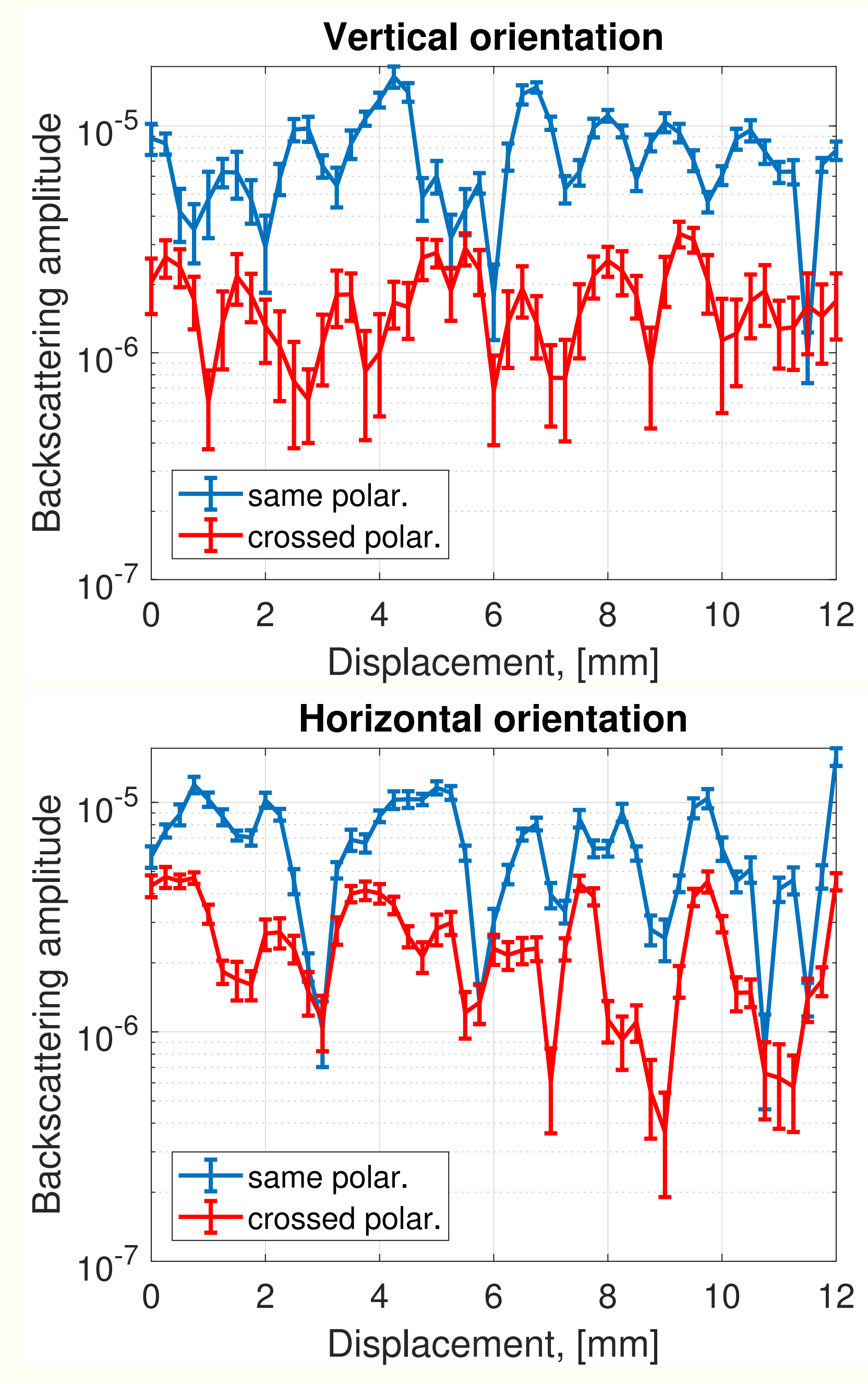

Figure 4: Crossed polarization of backscattered light is present and it is not negligible.

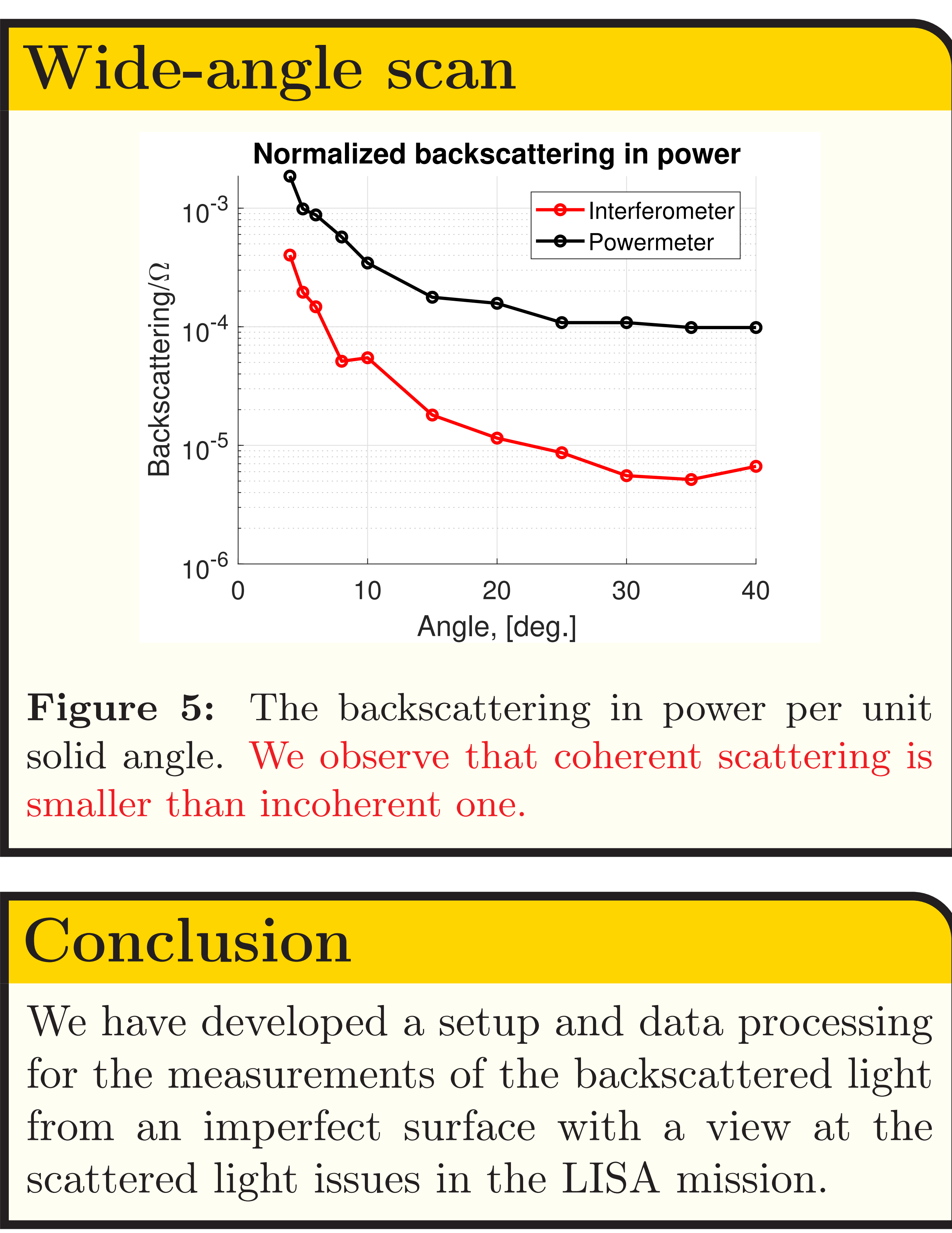

